\title{
Visual Perception of ADHD Children with Sensory Processing Disorder
}

\author{
Hyerim Jung ${ }^{1 *}$, Young Jae $\mathrm{Woo}^{2}$, Je Wook Kang ${ }^{3}$, Yeon Woo Choi ${ }^{4}$, and Kyeong Mi Kim ${ }^{5} \bowtie$ \\ 1Department of Rehabilitation Science, Graduate School of Inje University, Gimhae, Republic of Korea \\ ${ }^{2}$ Department of Psychiatry, Samsung Changwon Medical Center, Sungkyunkwan University, Seoul, Republic of Korea \\ ${ }^{3}$ Department of Psychiatry, Inje University Busan Paik Hospital, Busan, Republic of Korea \\ ${ }^{4}$ Department of Occupational Therapy, Graduate School of Inje University, Gimhae, Republic of Korea \\ ${ }^{5}$ Department of Occupational Therapy, College of Biomedical Science and Engineering, Inje University, Gimhae, Republic of Korea
}

\begin{abstract}
Objective The aim of the present study was to investigate the visual perception difference between ADHD children with and without sensory processing disorder, and the relationship between sensory processing and visual perception of the children with ADHD.

Methods Participants were 47 outpatients, aged 6-8 years, diagnosed with ADHD. After excluding those who met exclusion criteria, 38 subjects were clustered into two groups, ADHD children with and without sensory processing disorder (SPD), using SSP reported by their parents, then subjects completed K-DTVP-2. Spearman correlation analysis was run to determine the relationship between sensory processing and visual perception, and Mann-Whitney-U test was conducted to compare the K-DTVP-2 score of two groups respectively. Results The ADHD children with SPD performed inferiorly to ADHD children without SPD in the on 3 quotients of K-DTVP-2. The GVP of K-DTVP-2 score was related to Movement Sensitivity section $\left(\mathrm{r}=0.368^{*}\right)$ and Low Energy/Weak section of SSP ( $\left.\mathrm{r}=0.369^{*}\right)$.

Conclusion The result of the present study suggests that among children with ADHD, the visual perception is lower in those children with co-morbid SPD. Also, visual perception may be related to sensory processing, especially in the reactions of vestibular and proprioceptive senses. Regarding academic performance, it is necessary to consider how sensory processing issues affect visual perception in children with ADHD.

Psychiatry Investig 2014;11(2):119-123
\end{abstract}

Key Words Attention Deficit Disorder with Hyperactivity, Visual perception, Sensory processing disorder

\section{INTRODUCTION}

Children with attention deficit hyperactivity disorder (ADHD) experience difficulties because of the inattention, impulsivity and deficit of activity control that can have a severe effect on performance in school, home and community environment. ${ }^{1}$ Recently, several studies support the idea that individuals with ADHD have different visual perception. It was reported that a group with ADHD presented spatial bias

Received: March 8, 2013 Revised: April 24, 2013

Accepted: May 7, 2013 Available online: April 11, 2014

$\triangle$ Correspondence: Kyeong Mi Kim, PhD, OT

Department of Occupational Therapy, College of Biomedical Science and Engineering, Inje University, 197 Inje-ro, Gimhae 621-749, Republic of Korea

Tel: +82-55-320-3821, Fax: +82-55-326-4885

E-mail: kmik321@inje.ac.kr

*Current Affilation: Department of Occupational Therapy, Kaya University, Gimhae, Republic of Korea

(a) This is an Open Access article distributed under the terms of the Creative Commons Attribution Non-Commercial License (http://creativecommons.org/licenses/by$\mathrm{nc} / 3.0$ ) which permits unrestricted non-commercial use, distribution, and reproduction in any medium, provided the original work is properly cited. as part of impaired perceptual-attentional-action coordination. ${ }^{2}$ In multiple visual-spatial tasks intended for right hemisphere processing assessment in children from 14 to 16 years old, right hemisphere dysfunction was reported, and adults with ADHD were also impaired on a letter cancellation task. Teenagers and adults with ADHD demonstrated a higher mean left-sided omission rate than healthy controls. ${ }^{3,4}$ Visual perception problem in children with ADHD have been reported, however, little is known about the factors of visual perception in ADHD children.

Visual perception is viewed as an information-processing task involving the reception, organization, and assimilation of visual information in the central nervous system. ${ }^{5}$ If the information-processing centers of the brain do not run well, incomplete visual perception is the result. In several studies, hyper-reactivity to sensory stimuli in children with ADHD has been confirmed through parent-report and physiological testing, ${ }^{6}$ and they showed greater reactivity to sensory stimuli, with larger initial reactions and subsequent lack of habitua- 
tion, ${ }^{7}$ which is a natural tendency to diminish the distraction created by incoming sensory information. ${ }^{8}$ In addition, children with ADHD often suffer visual hypersensitivity or photophobia, diminished ability to process auditory or tactile input, and poor balance performance, equilibrium, postural control or coordination..$^{9-13}$ For these reasons, overactive and impulsive symptoms in sensory processing disorder can be easily confused with (and often co-occur with) ADHD, and children with ADHD often have comorbid sensory processing disorder. ${ }^{8}$ As a result, ADHD children who have sensory problems may also present different visual perception than children with $\mathrm{ADHD}$ who have typical sensory processing.

The main objective of the present study was to investigate the difference between children with ADHD with sensory processing disorder (SPD) and without SPD. It was hypothesized that children with ADHD and SPD would present with lower visual perception than children with ADHD without SPD, and sensory processing would be positively correlated with visual perception in ADHD.

\section{METHODS}

\section{Subjects}

Forty seven children who were psychiatric outpatients in Samsung Changwon Hospital from 2011 to 2012 participated in this study. They were diagnosed with ADHD by two psychiatrists, according to the criteria of the Diagnostic and Statistical Manual of Mental Disorders (DSM-IV-TR).

Children were excluded if: 1) Their IQ score were less than 80 on the KEDI-WISC, 2) had comorbid tic disorder, traumatic brain injuries, seizure disorder, or pervasive development disorder, or 3) had taken any psychiatric medication. A total of 38 children's data were analyzed and all 38 children were recruited. Children ranged from 6-8 years of age, and 34 (89.5\%) were boys and 4 (10.5\%) were girls. The total sample percentage distribution of children in each age level was as follows: 6 years old, 20.5\%; 7 years old, $50.5 \%$; 8 years old, $20 \%$, and their mean age were 7 years 3 months. The mean IQ was 103 .

\section{Procedure}

The present study was approved by the Institutional Review Board for human subjects at Sungkyunkwan University Samsung Changwon Hospital in Korea (2012-SCMC-008-00). Participants all completed Korean Wechsler Intelligence Scale for Children-III (K-WISC-III) and Korean Kiddie-Schedule for Affective Disorders and Schizophrenia-Present and Lifetime Version (K-SADS-PL). Subjects were asked to complete the Developmental Test of Visual Perception-2 (K-DTVP-2) by an occupational therapist, and parents completed Short Sensory profile (SSP). The subjects were clustered into two groups, based on SSP score. Twenty subjects with SSP score 154 or lower were clustered into SPD group, and the rest 18 subjects were clustered into non SPD (control) group.

\section{Measures}

\section{Korean Developmental Test of Visual Perception-2}

K-DTVP-2 is the Korean version of Developmental Test of Visual Perception-2. It evaluates children's visual perception. Based on eight separate but interrelated subscales: Eye-Hand Coordination, Copying, Spatial Relations, Position in Space, Figure-Ground, Visual Closure, Visual-Motor Speed, and Form Constancy.

The raw scores are recorded, then converted into standard scores, percentiles, and age equivalents using normative table. From the eight standard scores, two composite quotients are calculated, one regarding motor-reduced visual perception (MRP), and one regarding visual-motor integration (VMI). A general visual perception (GVP) composite quotient is also calculated by combining the standard scores from all eight of the subtests. ${ }^{14}$ In the present study, three composite quotient scores were analyzed: GVP, MRP, and VMI.

\section{Short Sensory Profile}

The Short Sensory Profile (SSP) ${ }^{15}$ is the shorter version of the Sensory Profile, ${ }^{16}$ which measures the child's sensory processing abilities, as expressed in the functional performance of daily life. SSP consists of 38 parent-reported items of behaviors associated with abnormal responses to sensory stimuli. The SSP yields a total score and seven section scores: Tactile Sensitivity, Taste/Smell Sensitivity, Movement Sensitivity, Under-responsive/seeks Sensation, Auditory Filtering, Low energy, and Visual/Auditory Sensitivity. Items are scored on Likert scale ( $1=$ always, $5=$ never). The possible range of raw scores on the total scale is 38-190, with higher scores (155-190) reflecting normal performance. A score of 142-154 reflects a probable difference in performance while a score of 38-141 reflects a definite difference in performance (Dunn, 1999). In the present study, SPD group was composed of subjects having a score of 38-154, and non SPD group was composed of subjects having a score of 155 and more. ${ }^{15}$

\section{Kiddie-Schedule for Affective Disorders and Schizophrenia-Present and Lifetime Version-Korean Version}

This is the Korean version of the K-SADS-PL, ${ }^{17}$ which is semi-structured parent interview and can be used for children from 6 years to 18 years of age. ${ }^{18}$ It takes about 1 hour 30 minutes to complete. Two psychiatrists administered interview in this research. 


\section{Statistical analysis}

In order to determine the relationship between sensory processing and visual perception, Spearman correlation analysis was run, and Mann-Whitney-U test was conducted to compare the K-DTVP-2 score (GVP, MRV and VMI) of two groups (SPD and non SPD) respectively.

$\mathrm{p}<0.05$ was considered significant. The Statistical Package for the Social Sciences (SPSS) version 19.0 was performed for Statistical analyses (version 19.0, SPSS Inc., Chicago, IL, USA).

\section{RESULTS}

\section{Comparison of group scores on SSP and K-DTVP-2}

The mean score for the SSP in the SPD group was 137.70, and in the non-SPD group was 167.80. The results of the Utest of K-DTVP-2 yielded a statistically significant result ( $\mathrm{p}=$ $0.012,0.033,0.026)$. The statistics for the individual factors are presented in Table 1 . The scores for the SPD group were significantly lower than the non SPD group on 3 quotients. This indicates that children with ADHD and SPD have diminished visual perception than those children without SPD.

\section{Relationship between sensory processing and visual perception}

The correlation coefficients between the total score and all scores of SSP and K-DTVP-2 of all subjects are presented in Table 2. Spearman correlation analysis indicates that a GVP of K-DTVP-2 score had a positive relationship with the total SSP score $\left(\mathrm{r}=0.334^{*}\right)$. The correlations between seven sections and three quotients of K-DTVP-2 were analyzed. The GVP score was positively related to Movement Sensitivity section $\left(r=0.368^{*}\right)$ and Low Energy/Weak section of SSP $\left(r=0.369^{*}\right)$.

\section{DISCUSSION}

It was hypothesized that children with ADHD and SPD would present with different visual perception than children with ADHD but without SPD, and that more accurate sensory processing would be correlated with more accurate visual perception in children with ADHD. The results of this study are consistent with these hypotheses.

The SPD group had lower mean scores on three quotients of K-DTVP-2 than the non SPD group, and the differences were statistically significant. We also observed that there was moderate relationship between general visual perception and sensory processing. There are several factors affecting visual perception, such as, attention, short- and long-term memory, individual's biases, feelings, attitudes, or life experiences. ${ }^{19-22}$ While sensory processing is only one of the factors influencing visual perception, a moderate correlation could be considered quite meaningful.

The finding indicates that two groups clustered into SPD and non SPD by sensory processing ability have different visual perceptions. What causes the visual perception difference between two groups of children with ADHD? The results reveal that, the VMI score on K-DTVP-2 of SPD group was significantly different from the score of the non-SPD group. Also, general visual perception related to the specific SSP subscales 'movement sensitivity' and 'low energy/weak.' 'Movement sensitivity' is described as over-responsiveness to vestibular stim-

Table 1. Comparison of group scores on SSP and K-DTVP-2

\begin{tabular}{|c|c|c|c|c|c|}
\hline & $\mathrm{SPD}(\mathrm{N}=20)$ & Non SPD $(\mathrm{N}=18)$ & Total subject $(\mathrm{N}=38)$ & Mann-Whitney-U & $\mathrm{p}$ \\
\hline \multicolumn{6}{|c|}{ K-DTVP-2 } \\
\hline GVP & $94.90(12.27)$ & $105.61(15.12)$ & $99.97(14.41)$ & 98 & 0.012 \\
\hline MRP & $95.05(16.27)$ & $106.61(15.97)$ & $100.52(16.58)$ & 107 & 0.033 \\
\hline VMI & $95.10(10.72)$ & $104.50(18.61)$ & $99.55(15.82)$ & 104 & 0.026 \\
\hline
\end{tabular}

Data presented as mean (SD). K-DTVP-2: Korean Developmental Test of Visual Perception-2, SSP: Short Sensory Profile, GVP: General visual perception, MRP: Motor reduced perception, VMI: Visual motor integration, N: number, SPD: sensory processing disorder

Table 2. Relationship between sensory processing and visual perception

\begin{tabular}{cccccccccc}
\hline & \multicolumn{7}{c}{ SSP } \\
\cline { 2 - 9 } & Total & TS & TSS & MS & USS & AF & LEW & VAS \\
\hline K-DTVP-2 & & & & & & & & \\
GVP & $0.334^{*}$ & 0.218 & 0.041 & $0.368^{*}$ & 0.263 & 0.254 & $0.369^{*}$ & 0.159 \\
MRP & 0.254 & 0.149 & -0.033 & 0.279 & 0.264 & 0.167 & 0.320 & 0.088 \\
VMI & 0.318 & 0.218 & 0.061 & 0.306 & 0.244 & 0.243 & 0.229 & 0.184 \\
\hline
\end{tabular}

Data presented as Spearman correlation coefficient. ${ }^{*} \mathrm{p}<0.05$. SSP: Short Sensory Profile, TS: Tactile Sensitivity, TSS: Taste/Smell Sensitivity, MS: Movement Sensitivity, USS: Underresponsive/Seeks Sensation, AF: Auditory Filtering, LEW: Low Energy/Weak, VAS: Visual/Auditory Sensitivity, K-DTVP-2: Korean Developmental Test of Visual Perception-2 
uli (e.g., 'dislikes activities where head is upside down', 'becomes anxious or distressed when feel leave the ground, and 'fears falling or heights'), and 'low energy/weak' is explained as a tendency to become tired and have weakness (e.g., 'tires easily, when standing or holding particular body position', 'poor endurance,' 'seems to have weak muscles'). The results reflect that decreased sensory processing in these areas may be a hallmark of children with ADHD who have visual perception problems, and might indicate such when compared to typically-developing children. In a previous study, ADHD group displayed distinguishable 'low energy/weak' compared to typical children in SSP. ${ }^{23}$ In addition, other studies suggested developmental coordination disorder (dyspraxia) often cooccurs with $\mathrm{ADHD},{ }^{8}$ and more than one third of children with $\mathrm{ADHD}$ have poor balance and coordination ${ }^{11}$ associated with sensory inputs, sensory integration, and/or the inhibition of excessive movements. ${ }^{24}$ Children with ADHD often have vestibular or proprioceptive problems which may be related to decreased visual perception.

Vestibular input and proprioception from eyes, neck and body, and visual information are integrated to form a "map" that is used to "navigate" the body successfully in space, they are unified into one composite sensory process in the brain stem. This sensory process helps us to direct our eyes when we look at things. If vestibular and proprioceptive sensations are not properly organized to keep the eyes moving smoothly, the child may suffer great discomfort when reading. ${ }^{25}$ Therefore, children with ADHD and sensory processing disorder who suffer vestibular or proprioception process problems have deficits of focusing on an object to provide visual information, and of maintaining their posture to perform tabletop activities such as writing or reading. Also children whose major problem is in processing vestibular input may also score low in visual perception tests. ${ }^{25}$

Visual perceptual problems can impact many areas of activities of daily living, especially school performance, for children. Sensory processing which affects several factors related to visual perception is essential performing visual-processing tasks such as matching shapes or objects, or tasks containing unfamiliar stimuli or subtle discriminations. Children may experience difficulty in recognizing similar letters or words, and may reverse letters and numbers in spelling. They may not be able to interpret charts, maps, graphs, symbols, and diagrams. In addition, activities that require fine motor and gross motor skills, such as handwriting and left/right discrimination can also be affected by visual perceptual problems. ${ }^{26} \mathrm{How}$ ever, visual perception is easily overlooked, while diagnosis and assessment are focused only on 1 more obvious dysfunctions in children with ADHD. Professionals may be overlooking a very important issue for children with ADHD. Without a vi- sual perception assessment perceiving how they process visual information in their central nervous system, it is much more difficult to understand and help their daily activities such as performance in school. Sensory processing's effects on the visual perception of children with ADHD.

In summary, our findings confirmed the influence of sensory processing on visual perception in children with ADHD. The findings indicate that different visual perceptions do exist between children with ADHD with and without SPD. Also, sensory processing, especially vestibular and proprioceptive processing, is related to the visual perception among children with ADHD. This research can help to categorize potential difficulties of visual perception dysfunctions in children with ADHD. The limitations of this study are 1) control group of normal children was not involved, so it could not investigate the visual perception differences between ADHD children and normal children, however, through normative referencing, these children may be compared to their peers; 2 ) it had an insufficient number of subjects to examine parametric data analysis. However, the exclusion criteria of this study helped to strengthen its rigor; selecting children with $\mathrm{ADHD}$ without confounding factors such as medication adjustment allowed for a more accurate generalization of the results.

It is recommended that future works need to analyze sensory processing as a factor that affects the visual perception of children with ADHD, and to exam the effects of vestibular and proprioceptive senses related to movement and posture on visual perception. Using a greater number of participants in order to provide theoretical refinement of the impact of sensory processing on children with ADHD would be helpful. Also, investigating the correlations between sensory processing and visual perception for children with diverse diagnosis is required.

\section{Acknowledgments}

This work was supported by the 2011 Inje University research grant.

\section{REFERENCES}

1. American Psychiatric Association. Diagnostic and Statistical Manual of Mental Disorders, Fourth Edition. Washington DC: American Psychiatric Association; 1994.

2. Kelly A, Uddin LQ, Biswal BB, Castellanos FX, Milham MP. Competition between functional brain networks mediates behavioral variability. Neuroimage 2008;39:527-537.

3. Garcia-Sanchez C, Estevez-Gonzalez A, Suarez-Romero E, Junque C. Right hemisphere dysfunction in subjects with attention-deficit disorder with and without hyperactivity. J Child Neurol 1997;12:107-115.

4. Sandson TA, Bachna KJ, Morin MD. Right Hemisphere Dysfunction in ADHD: visual hemispatial inattention and clinical subtype. J Learn Disabil 2000;33:83-90.

5. Toglia JP. Visual perception of objects: an approach to assessment and intervention. Am J Occup Ther 1989;43:587-595.

6. Reynolds S, Lane SJ. Diagnostic validity of sensory over-responsivity: a review of the literature and case reports. J Autism Dev Disord 2008; 
38:516-529.

7. Mangeot SD, Miller LJ, McIntosh DN, McGrath-Clarke J, Simon J, Hagerman RJ, et al. Sensory modulation dysfunction in children with attention-deficit-hyperactivity disorder. Dev Med Child Neurol 2001; 43:399-406.

8. Miller LJ, Anzalone ME, Lane SJ, Cermak SA, Osten ET. Concept evolution in sensory integration: a proposed nosology for diagnosis. Am J Occup Ther 2007;61:135-140.

9. Cheung PPP, Siu AM. A comparison of patterns of sensory processing in children with and without developmental disabilities. Res Dev Disabil 2009;30:1468-1480.

10. Hern KL, Hynd GW. Clinical differentiation of the attention deficit disorder subtypes: Do sensorimotor deficits characterize children with ADD/WO? Arch Clin Neuropsychol 1992;7:77-83.

11. Sergeant JA, Piek JP, Oosterlaan J. ADHD and DCD: a relationship in need of research. Hum Mov Sci 2006;25:76-89.

12. Ghanizadeh A, Aghakhani K. Photophobia and methylphenidate. Psychopharmacol Bull 2008;41:171-173.

13. Ghanizadeh A. Visual fields in children with attention-deficit/hyperactivity disorder before and after treatment with stimulants. Acta Ophthalmol 2010;88:e56.

14. Brown T, Rodger S, Davis A. Factor structure of the four motor-free scales of the Developmental Test of Visual Perception, (DTVP-2). Am J Occup Ther 2008;62:502-513.

15. McIntosh D, Miller LJ, Shyu V. Development and Validation of the Short Sensory Profile. In: Dunn W, editor. Sensory Profile: Examiner's Manual. San Antonio, TX: Psychological Corporation; 1999.

16. Dunn W. The Sensory Profile: Examiner's Manual. San Antonio, TX: Psychological Corporation; 1999.
17. Kaufman J, Birmaher B, Brent D, Rao U, Flynn C, Moreci P, et al. Schedule for Affective Disorders and Schizophrenia for School-Age Children-Present and Lifetime Version (K-SADS-PL): initial reliability and validity data. J Am Acad Child Adolesc Psychiatry 1997;36:980-988.

18. Kim YS, Cheon KA, Kim BN, Chang SA, Yoo HJ, Kim JW, et al. The reliability and validity of Kiddie-schedule for affective disorders and schizophrenia-present and lifetime version-Korean version (K-SADSPL-K). Yonsei Med J 2004;45:81-89.

19. Rock I, Linnett CM, Grant P, Mack A. Perception without attention: results of a new method. Cognit Psychol 1992;24:502-534.

20. Kappas A, Olk B. The concept of visual competence as seen from the perspective of the psychological and brain sciences. Visual Stud 2008; 23:162-173.

21. Segall MH, Campbell DT, Herskovits MJ. The Influence of Culture on visual Perception. Indianapolis: Bobbs-Merrill Co; 1966.

22. Vygotskil LS, Cole M. Mind in Society: The Development of Higher Psychological Processes. Cambridge, MA: Harvard University Press; 1978.

23. Roley SS, Blanche EI, Schaaf RC. Understanding the Nature of Sensory Integration with Diverse Populations. San Antonio: Therapy Skill Builders; 2001.

24. Zang Y, Gu B, Qian Q, Wang Y. Objective measurement of the balance dysfunction in attention deficit hyperactivity disorder children. Chin J Clin Rehabil 2002;6:1372-1374.

25. Ayres AJ, Robbins J. Sensory Integration and the Child: Understanding hidden Sensory Challenges. Los Angeles: Western Psychological Service; 2005.

26. Ayres AJ. Types of sensory integrative dysfunction among disabled learners. Am J Occup Ther 1972;26:13-18. 